Supporting information

\title{
Adapting a Fourier-transform ion cyclotron resonance mass spectrometer for gas-phase fluorescence spectroscopy of trapped biomolecular ions
}

Ri Wu, Jonas B. Metternich, Prince Tiwari, and Renato Zenobi*

Department of Chemistry and Applied Biosciences, ETH Zürich

Vladimir-Prelog-Weg 3, 8093 Zürich (Switzerland)

*Corresponding author’s email: zenobi@org.chem.ethz.ch 


\section{Table of Content}

Scheme S1. The laser and fluorescence detection system of the modified FT-ICR mass spectrometer. Laser and fluorescence detection devices are shared with another instrument, and this scheme was adapted from earlier publications from our group. ${ }^{3,4}$ Solid lines, mirrors; dashed lines, flippable mirrors; NDF, neutral density filter; BB, beam blocker; L1-L6, lens 1-6; PH1-2, pinholes 1-2; S, shutter.

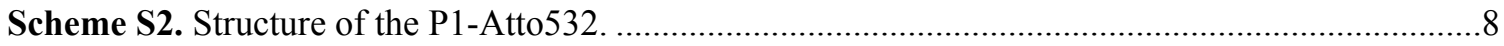

Figure S1. (a-c) Mass deviation of NaTFA peaks before calibration and fitting with a 2nd-order polynomial function.. (d-f) Mass error after quadratic calibration. Mass spectra of different transient (FID) length $(50 \mathrm{~ms}, 100 \mathrm{~ms}$, and $200 \mathrm{~ms})$ were acquired and its average mass error are $37.1 \mathrm{ppm}, 13.2 \mathrm{ppm}$, and $6.1 \mathrm{ppm}$. Sequential mode was applied, with accumulation ending at 1.0 $\mathrm{s}$, excitation starting at $2.0 \mathrm{~s}$, and signal processing for another $0.5 \mathrm{~s}$ to $3.0 \mathrm{~s}$.

Figure S2. The mass spectral signal intensity of Rh6G cations with a different MS accumulation time.

Figure S3. The user interface of the new DAQ system, with a similar design and operation to ftmsControl 2.1 (Bruker Daltonics).

Figure S4. Fluorescence signal intensity under the effect of (a) laser power and (b) irradiation time. CCD accumulation time was $300 \mathrm{~s}$. The total fluorescence collection time was $180 \mathrm{~s}$. A $3 \mathrm{~s}$ laser irradiation time and $10 \mathrm{~mW}$ laser power were applied for this investigation. The excitation wavelength $\left(\lambda_{\text {ex }}\right)$ was $480 \mathrm{~nm}$. The sequential mode was adopted. The fluorescence intensity increment curve was calculated after normalization of MS signal intensity and duty cycle. Normalized PD yield is defined as the ratio of the most abundant fragment ion (i.e., [M-CO] $415.31 \mathrm{Th}$ ) to the precursor ion

Figure S5. Mass spectra of ubiquitin-CR6G after in-cell isolation, (a) $6+$, and (b) $5+\ldots \ldots \ldots \ldots \ldots \ldots \ldots \ldots . . . .8$

Figure S6. PD mass spectra of (a) Rh6G after being irradiated under $10 \mathrm{~mW}$, and (b) P1-Atto 532 labeled peptide after being irradiated under $62.3 \mathrm{~mW}$. The wavelength of the laser was $480 \mathrm{~nm}$, and irradiance lasted for $2.95 \mathrm{~s}$

Table S1. Conditions of the gas-phase fluorescence experiments for different ions. . .8

Table S2. List of the working conditions in FT-ICR MS 
Materials. Rhodamine 110 (Rh110), rhodamine 6G (Rh6G), and rhodamine B (RhB) were purchased from Acros Organics (Liege Area, Belgium). Atto 532 maleimide dye was purchased from Atto-Tec (Siegen, Germany). Carboxyrhodamine 6G (CR6G) succinimidyl ester was purchased from AnaSpec (California, USA). Lyophilized powders of ubiquitin (Ubi) and apomyoglobin (apoMb) were obtained from SigmaAldrich (St. Louis, USA). P1 (CAAAAHAAAHAAAAHAAAHAAACAK) was purchased from GenScript (Leiden, Netherland), with acetylation and amidation for $\mathrm{N}$ - and C-terminal. The dyes were dissolved in HPLC grade solvents (water or methanol) and diluted to a concentration of $10 \mu \mathrm{M}$. The labeling was performed according to an established protocol. ${ }^{1}$ Briefly, the peptide powder or lyophilized protein was dissolved in $0.1 \mathrm{M}$ potassium phosphate buffer $(\mathrm{pH}=8.3$ for NHS ester, $\mathrm{pH}=7.1$ for maleimide) at a concentration of approximately $5 \mathrm{mg} / \mathrm{mL}$, and reactive dye was dissolved in DMSO or DMF $(10 \mathrm{mg} / \mathrm{mL})$. Reactive dye solution ( $20 \mu \mathrm{L}$ ) was added to the sample solution, slowly vortexed, and incubated for 1 hour with ice cooling. Finally, the sample was purified using an HPLC equipped with a UV/Vis detector. Fully/partially separated components were collected and then lyophilized. Aliquots of the samples were analyzed by mass spectrometry to verify the identity of the labeling products. The dye-labeled biomolecules were dissolved in water and analysed by UV/VIS spectroscopy at $522 \mathrm{~nm}$ to determine the quantity. Then samples were lyophilized again for storage. Before analysis, the samples were dissolved in water, and the concentration was adjusted to $\sim 10 \mu \mathrm{M}$. To label cysteine groups with maleimide dyes, a reduction of the cysteine residue was performed using dithiothreitol (DTT). The excess DTT was removed by HPLC purification and the peptide lyophilized.

Laser system and fluorescence detection. The laser and fluorescence detection devices used in this work have been described in detail elsewhere. ${ }^{3}$ The schematic of the experimental setup is also presented in scheme S1. Briefly, an $80 \mathrm{MHz}$ tunable femtosecond pulsed Ti: Sapphire laser generates $<100 \mathrm{fs}$ pulses in the 690-1040 nm range. A second harmonic generator (UHG, Spectra-Physics, California, USA) doubles the frequency for an output of 345-520 $\mathrm{nm}$ with a power of $\sim 0.08-1.16 \mathrm{~W}$. After passing through a polarizer, one pulse out of three is picked using a pulse picker (350-105-UV, Conoptics, Danbury, USA) to allow $\sim 37.5$ ns for fluorescence lifetime experiments. The pulse picker was bypassed in other experiments. A continuously variable neutral density filter (NDC-50C-4M, Thorlabs) is used to adjust the laser power. A laser shutter could also be installed and triggered to irradiate the ion cloud while trapped in the ICR cell. To decrease the diameter of the laser beam, a lens (L3, $f=750 \mathrm{~mm}, \mathrm{~d}=25.4 \mathrm{~mm}, \mathrm{LB} 1475-\mathrm{A}$, Thorlabs) was installed in the beam path at $\sim 750 \mathrm{~mm}$ from the center of the ICR cell. When the trapped ions are irradiated 
with a laser beam of appropriate wavelength and power, fluorescence is emitted. $\sim 10 \%$ of it ( $\sim 2 \%$ previously) is collected through the lens $(\mathrm{L} 1, f=25 \mathrm{~mm}, \mathrm{~d}=38.1 \mathrm{~mm})$, further collimated with another lens (L2, $f=50 \mathrm{~mm}$, $\mathrm{d}=40 \mathrm{~mm}$ ), and out of the MS through a viewport. The light was focused onto a $10 \mathrm{~m}$-long $3 \mathrm{~mm}$ o.d. plastic fiber (NA=0.51, PFR FB 3000, SEDI), which was then connected to a lens ( $\mathrm{L} 4, \mathrm{f}=50 \mathrm{mmm}, \mathrm{d}=25.4 \mathrm{~mm}$, AL2550G-A, Thorlabs) for collimation, and a long pass filter (442, 473, 496 or $514 \mathrm{~nm}$, AHF analysentechnik) was used to eliminate the laser background. $\sim 2 \%$ of emitted fluorescence is eventually transferred due to losses in all the optical elements. The fluorescence detection setup consists of a spectrograph (Kymera 193i, Oxford Instruments, Abingdon, UK) coupled to a CCD camera (Andor Newton 920, Oxford Instruments, Abingdon, UK) or alternatively a single-photon avalanche photodiode (SPAD, MPD-100-CTB, Picoquant, Berlin, Germany) for fluorescence lifetime experiments. A replaceable 2-inch mirror can also direct the beam to be either focused onto the entrance slit of the spectrograph $(\sim 3.0 \mathrm{~mm}$ width) or the active area of the SPAD by plano-convex lenses (L5 \& L6, f=50 mm, d=25.4 mm, LA4148, Thorlabs). 


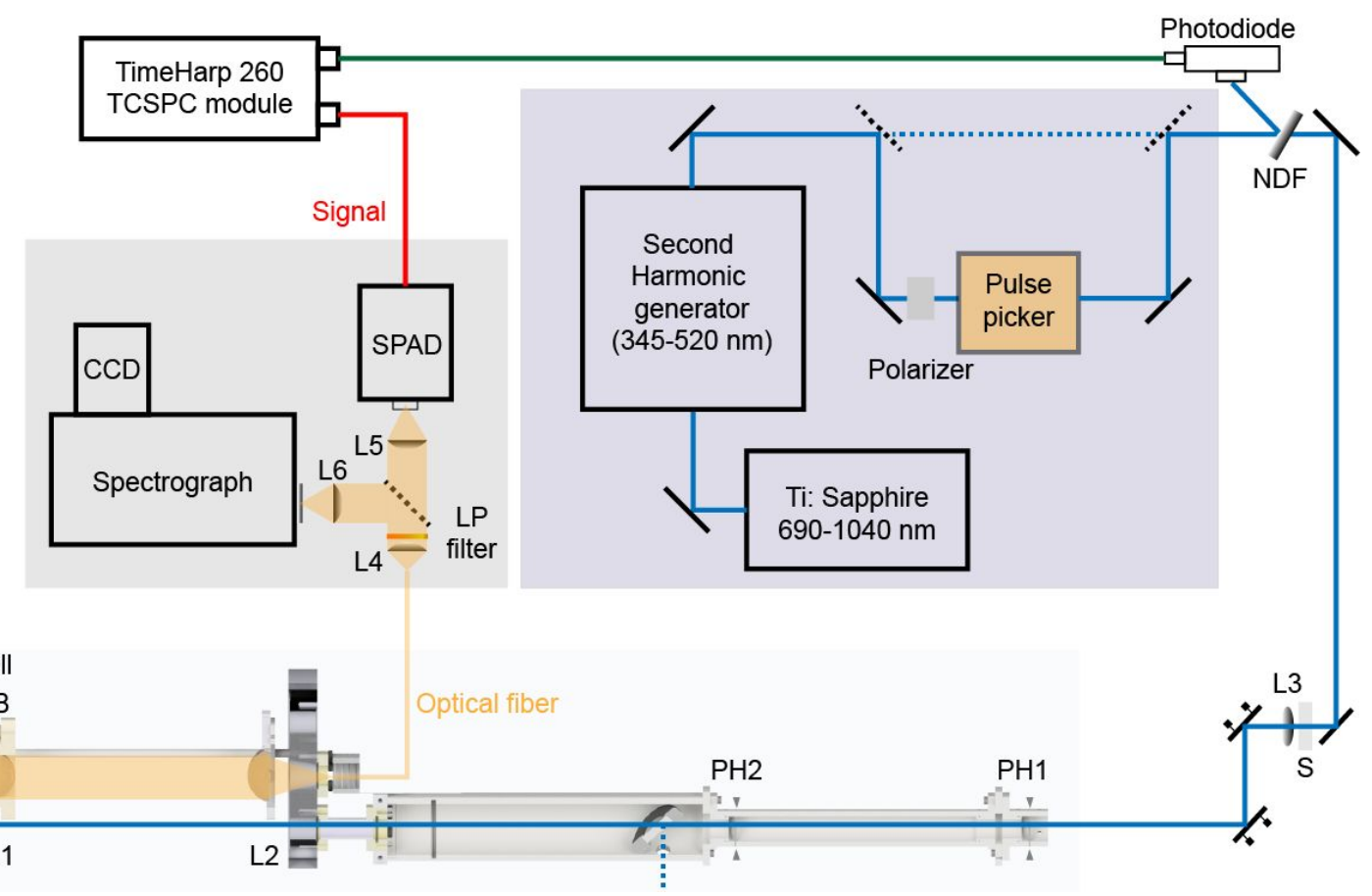

Scheme S1. The laser and fluorescence detection system of the modified FT-ICR mass spectrometer. Laser and fluorescence detection devices are shared with another instrument, and this scheme was adapted from earlier publications from our group. ${ }^{3,4}$ Solid lines, mirrors; dashed lines, flippable mirrors; NDF, neutral density filter; BB, beam blocker; L1-L6, lens 1-6; PH1-2, pinholes 1-2; S, shutter. 
a

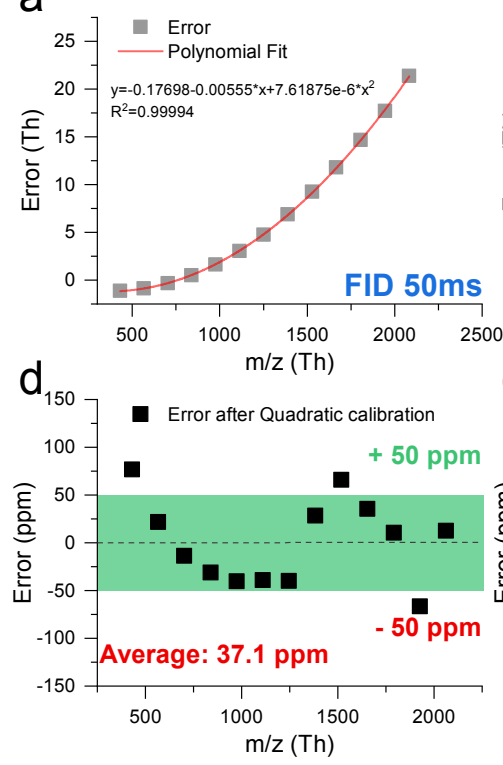

b
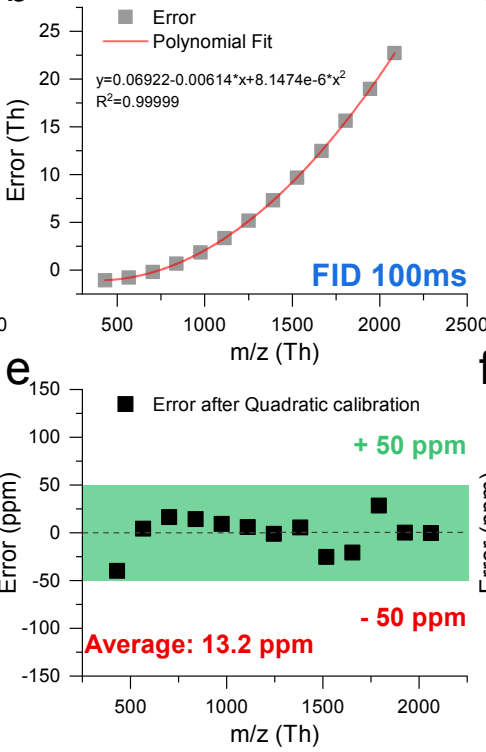

C
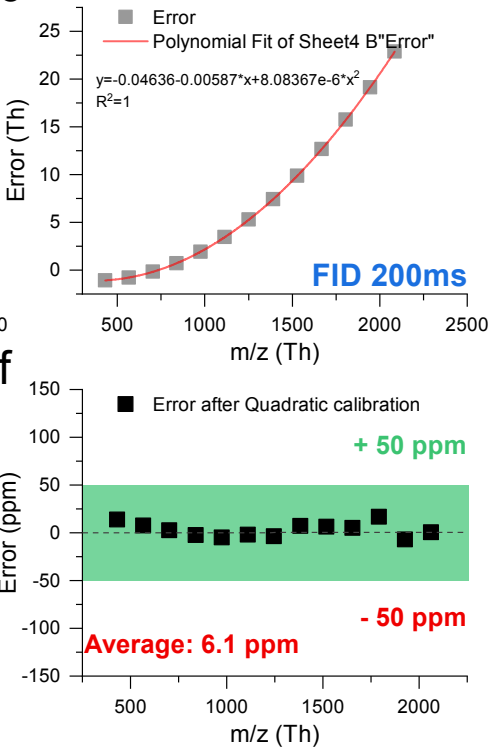

Figure S1. (a-c) Mass deviation of NaTFA peaks before calibration and fitting with a 2nd-order polynomial function.. (d-f) Mass error after quadratic calibration. Mass spectra of different transient (FID) length (50 ms, $100 \mathrm{~ms}$, and $200 \mathrm{~ms}$ ) were acquired and its average mass error are $37.1 \mathrm{ppm}, 13.2 \mathrm{ppm}$, and $6.1 \mathrm{ppm}$. Sequential mode was applied, with accumulation ending at $1.0 \mathrm{~s}$, excitation starting at $2.0 \mathrm{~s}$, and signal processing for another $0.5 \mathrm{~s}$ to $3.0 \mathrm{~s}$.

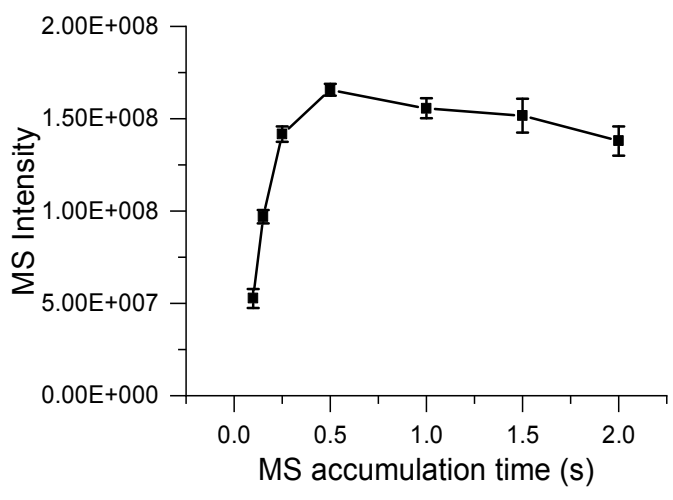

Figure S2. The mass spectral signal intensity of Rh6G cations with a different MS accumulation time. 


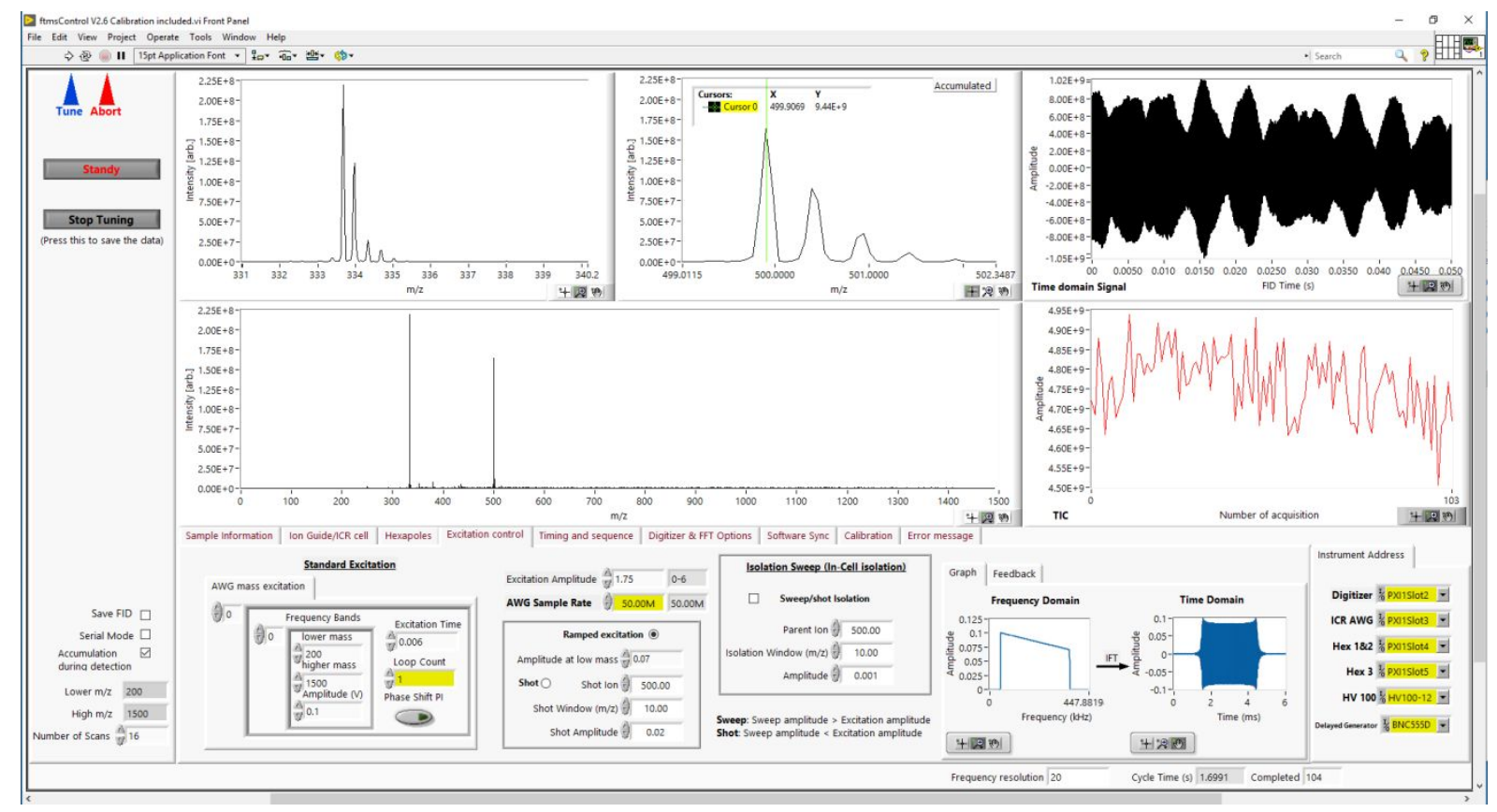

Figure S3. The user interface of the new DAQ system, with a similar design and operation to ftmsControl 2.1 (Bruker Daltonics).
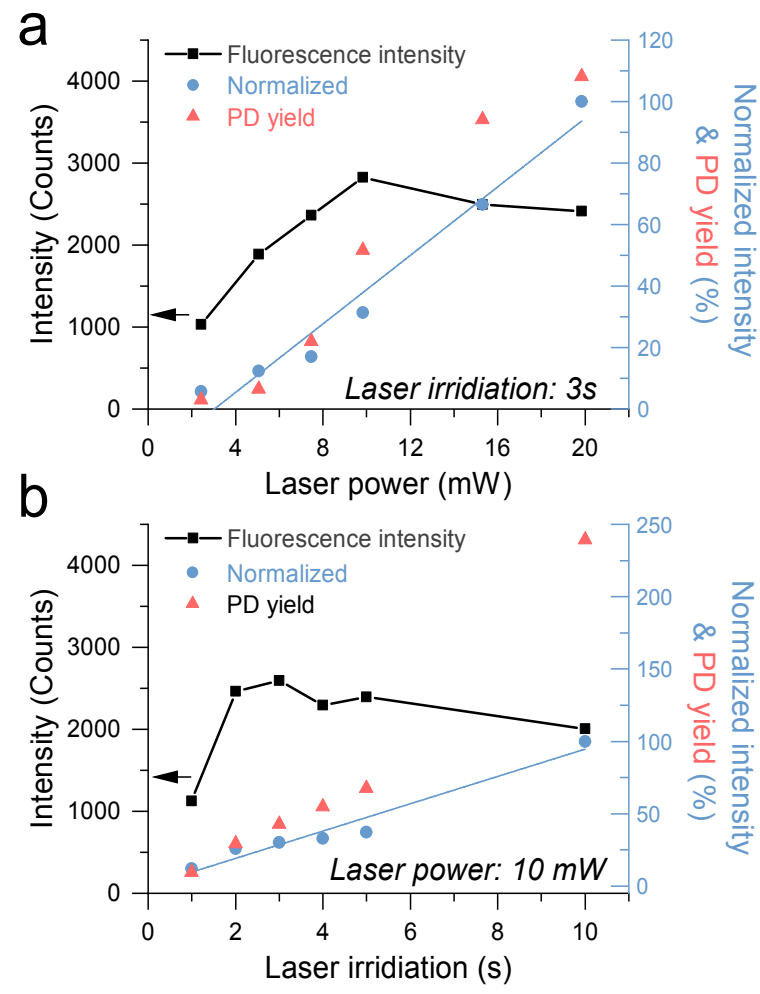

Figure S4. Fluorescence signal intensity under the effect of (a) laser power and (b) irradiation time. CCD accumulation time was $300 \mathrm{~s}$. The total fluorescence collection time was $180 \mathrm{~s}$. A $3 \mathrm{~s}$ laser irradiation time and $10 \mathrm{~mW}$ laser power were applied for this investigation. The excitation wavelength $\left(\lambda_{\mathrm{ex}}\right)$ was $480 \mathrm{~nm}$. The 
sequential mode was adopted. The fluorescence intensity increment curve was calculated after normalization of MS signal intensity and duty cycle. Normalized PD yield is defined as the ratio of the most abundant fragment ion (i.e., $[\mathrm{M}-\mathrm{CO}]^{+} 415.31 \mathrm{Th}$ ) to the precursor ion.

Table S1. Conditions of the gas-phase fluorescence experiments for different ions.

\begin{tabular}{cccccc}
\hline Ions & $\begin{array}{c}\boldsymbol{\lambda}_{\text {ex }} \\
(\mathbf{n m})\end{array}$ & $\mathbf{P}(\mathbf{m W})$ & $\begin{array}{c}\text { Fluorescence collection } \\
\left(\mathbf{T}_{\mathbf{f}}, \mathbf{s}\right)\end{array}$ & $\begin{array}{c}\text { Ion intensity } \\
\left(\mathbf{1 0}^{\mathbf{8}}\right)\end{array}$ & Counts \\
\hline Rh6G (1+) & 480 & 10 & 180 & 5.4 & 1897 \\
Rh110 (1+) & 430 & 10 & 180 & 3.0 & 955 \\
RhB (1+) & 480 & 10 & 600 & 3.4 & 1465 \\
P1-Atto 532 (3+) & 500 & 10 & 600 & 8.4 & 728 \\
P1-Atto 532 (4+) & 500 & 10 & 600 & 5.9 & 985 \\
Ubi-CR6G (6+) & 480 & 10 & 600 & 6.0 & 1009 \\
apoMb-CR6G (15+) & 490 & 10 & 600 & 0.5 & 570 \\
\hline
\end{tabular}

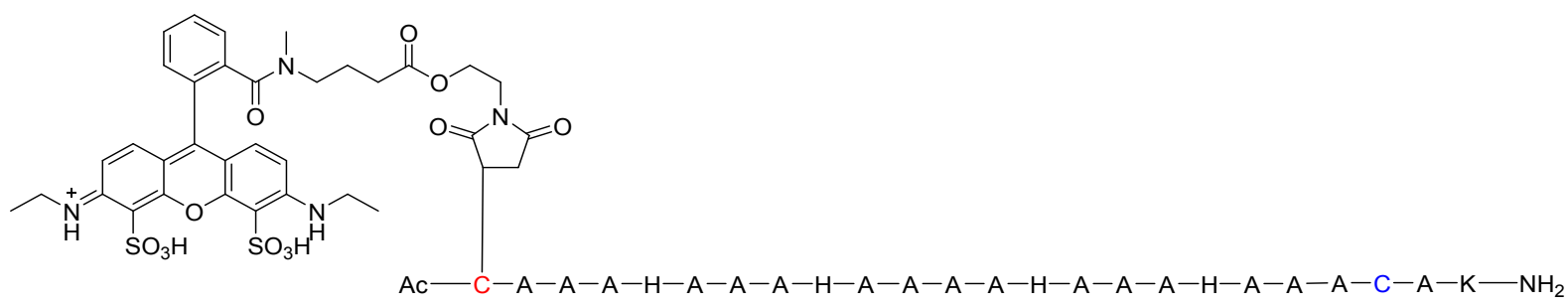

Scheme S2. Structure of the P1-Atto532.
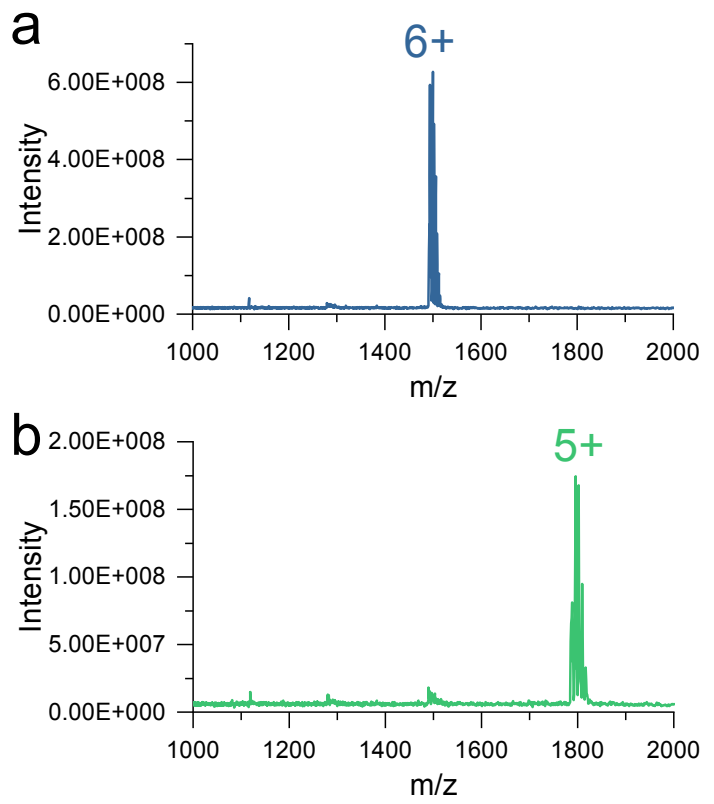

Figure S5. Mass spectra of ubiquitin-CR6G after in-cell isolation, (a) 6+, and (b) $5+$. 


\section{a}

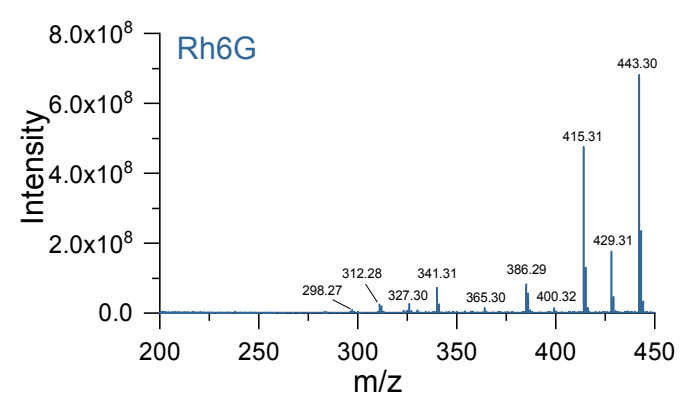

b

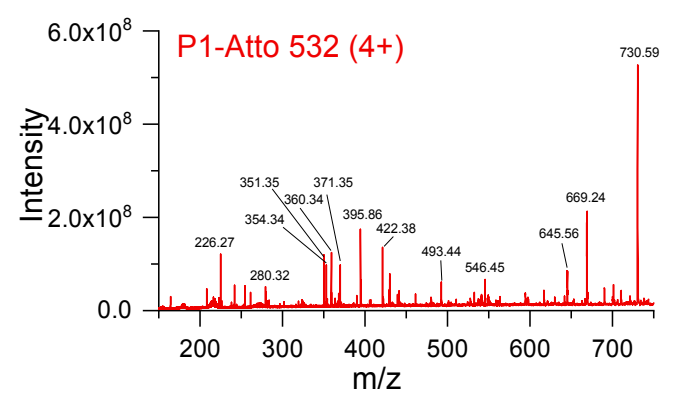

Figure S6. PD mass spectra of (a) Rh6G after being irradiated under $10 \mathrm{~mW}$, and (b) P1-Atto 532 labeled peptide after being irradiated under $62.3 \mathrm{~mW}$. The wavelength of the laser was $480 \mathrm{~nm}$, and irradiance lasted for $2.95 \mathrm{~s}$.

Table S2. List of the working conditions in FT-ICR MS.

\begin{tabular}{|c|c|c|c|}
\hline Parameter & Value & Parameter & Value \\
\hline Low m/z (Th) & $50-300$ & $\mathrm{Hex}_{1} \& \mathrm{Hex}_{2} \mathrm{RF}$ frequency & $1 \mathrm{MHz} / 250 \mathrm{kHz}$ \\
\hline $\operatorname{High} \mathrm{m} / \mathrm{z}(\mathrm{Th})$ & $1000-5000$ & $\operatorname{Hex}_{1} \& \operatorname{Hex}_{2} R F$ amplitude $\left(V_{p-p}\right)$ & $0.0-420.0 / 0.0-700.0$ \\
\hline \multirow[t]{2}{*}{ Number of Scans } & $8-32$ & $\mathrm{Hex}_{3}$ frequency & $2.51 \mathrm{MHz} / 0.777 \mathrm{MHz}$ \\
\hline & & $\operatorname{Hex}_{3} \mathrm{RF}$ amplitude $\left(\mathrm{V}_{\mathrm{p}-\mathrm{p}}\right)$ & $0.0-300.0 / 0.0-450.0$ \\
\hline Sample cone (V) & $30.0-100.0$ & Excitation time (ms) & 6.0 \\
\hline Extractor cone $(\mathrm{V})$ & $7.0-9.0$ & Excitation amplitude $\left(\mathrm{V}_{\mathrm{p}-\mathrm{p}}\right)$ & $0-300.0$ \\
\hline $\mathrm{Hex}_{1}$ DC offset (V) & $5.0-7.0$ & & ADD / Sequential mode \\
\hline $\operatorname{Hex}_{2}$ entrance $(V)$ & $4.5-6.0$ & Ion jection time (ms) & $1000 / 1000$ \\
\hline $\mathrm{Hex}_{2}$ DC offset (V) & $0.0-5.0$ & ICR starting trigger $(\mathrm{ms})$ & $800 / 4000$ \\
\hline $\mathrm{Hex}_{2}$ exit high (V) & $5.0-20.0$ & Cycle time (ms) & $3200 / 4500$ \\
\hline $\mathrm{Hex}_{2}$ exit low (V) & $-30.0-0.0$ & $\mathrm{Hex}_{3} \mathrm{RF}$ start time (ms) & 900 \\
\hline Shutter DC (V) & -10.0 & $\mathrm{Hex}_{3} \mathrm{RF}$ end time (ms) & 1010 \\
\hline Hex lense (V) & 0.0 & Shutter pulse start time (ms) & 900 \\
\hline $\mathrm{Hex}_{3}$ DC offset (V) & -10.0 & Shutter pulse duration (ms) & 110 \\
\hline Front trap plate (V) & $5.0-20.0$ & Ion flight time (ms) & $0.5-5.0$ \\
\hline Back trap plate $(\mathrm{V})$ & $5.0-20.0$ & Transient length (ms) & 50 \\
\hline
\end{tabular}

\section{References:}

(1) Thermofisher. Amine-Reactive Probes | 2 Labeling Proteins. https://tools.thermofisher.com/content/sfs/manuals. 
(2) Czar, M. F.; Zosel, F.; König, I.; Nettels, D.; Wunderlich, B.; Schuler, B.; Zarrine-Afsar, A.; Jockusch, R. A. Gas-Phase FRET Efficiency Measurements to Probe the Conformation of MassSelected Proteins. Anal. Chem. 2015, 87 (15), 7559-7565.

(3) Tiwari, P.; Metternich, J. B.; Czar, M. F.; Zenobi, R. Breaking the Brightness Barrier: Design and Characterization of a Selected-Ion Fluorescence Measurement Setup with High Optical Detection Efficiency. J. Am. Soc. Mass Spectrom. 2021, 32 (1), 187-197.

(4) Czar, M. F.; Tiwari, P.; Zenobi, R. Towards Fluorescence-Based Probes of Gas-Phase Protein Structure. SCS Fall Meet. [Conference Present. 2017, Bern, Switzerland, http://fm17.chemistrycongresses.ch/. 